\title{
HISTORIA DE LA ESCUELA UNIVERSITARIA DE LAS CIENCIAS DE LA SALUD
}

\author{
History of the Universitary School of Health Sciences
}

\author{
*José Raúl Arita Chávez.
}

\section{INTRODUCCIÓN}

La Universidad Nacional Autónoma de Honduras (UNAH) en el marco de la reforma universitaria crea la Escuela Universitaria de las Ciencias de la Salud (EUCS) en la UNAH del Valle de Sula, para responder a las necesidades de formación los profesionales de ciencias de la salud con competencias de desempeño en equipo, con enfoque social, ético y humanístico; mediante la integración de las carreras de Enfermería, Odontología y Medicina a nivel de grado, así como los postgrados de Pediatría y Gineco-Obstetricia. Entre las aportaciones y avances de la EUCS, podemos mencionar: a) Actividades de vinculación constantemente con la sociedad local y en general en los municipios de la Zona Metropolitana del Valle de Sula, b) Fortalecimiento de los procesos académico-administrativos através de una estructura organizativa funcional e interdisciplinaria que se ha consolidado paulatinamente c) Se ha estimulado la ejecución de proyectos de investigación que son desarrollados por estudiantes, docentes y grupos inter y multidisciplinarios, divulgados en diferentes revistas nacionales e internacionales y principalmente en la Revista Científica de la Escuela Universitaria de las Ciencias de la Salud y la revista Acta Pediátrica Hondureña, d) Se ha avanzado significativamente en infraestructura y equipo, próximos a inaugurar el complejo de ciencias de la salud que estará equipado con laboratorios modernos donde realizarán sus prácticas los estudiantes de pregrado, grado y posgrado. Todo se está logrando con el apoyo de las autoridades y el desempeño de una planta de docentes calificados y cuantificados que con ímpetu y alto sentido de pertenencia, se enfocan y aportan a la misión y visión de la EUCS.

*Director de la Escuela Universitaria de las Ciencias de la Salud de la UNAH-VS, Médico y Cirujano, Master en Educación Superior.

Dirigir correspondencia a: jaritac@unah.edu.hn

Recibido: 15 de junio 2016,

Aprobado: 30 de junio 2016

\section{DESARROLLO DEL TEMA}

La UNAH para dar respuesta a las necesidades de salud de la población hondureña, creó en el Centro Universitario Regional del Norte (CURN), ahora Universidad Nacional Autónoma de Honduras en el Valle de Sula (UNAH-VS); la Carrera de Enfermería en el año 1979, Odontología en el año 2000, Medicina en el año 2003 y los postgrados de Pediatría y Gineco-obstetricia el 2006. La Institución consideró urgente formar profesionales con capacidad de trabajar en equipo y con visión social del problema de salud. ${ }^{(1)}$

La idea de crear la Escuela Universitaria de las Ciencias de la Salud (EUCS) nace en 2006, año en que se procedió a la construcción colectiva de la misión y visión de la EUCS como nueva unidad académica para la integración de los diferentes departamentos y para viabilizar el proyecto de creación de la EUCS, se realizó un foro con representantes de la Secretaría de Salud y de Colegios Profesionales de las Ciencias de la Salud y las Ciencias Sociales, a quienes se les presentó la idea, propuesta que tuvo buena aceptación. Para dinamizar el proyecto, el Presidente de la Comisión de Transición, Dr. Jorge Haddad Quiñónez, y la Dra. Norma Martín de Reyes, también miembro de la Comisión, en compañía de la Vice-Rectora Académica, Dra. Rutilia Calderón, consolidaron el Grupo Gestor de la EUCS en reunión realizada ese mismo año. ${ }^{(2)}$

En diciembre del 2006 se realizó jornada de trabajo con la Dra. María del Carmen Amaro Cano como consultora internacional cubana y el grupo gestor ampliado para abordar los temas de ética y promoción de la salud como eje curricular de tal manera que durante el primer trimestre se trabajó la fundamentación curricular para la EUCS. 
En febrero del 2007 se integró a médicos docentes de ciencias morfológicas, fisiológicas, de odontología, y de medicina para conformar posteriormente el departamento de Ciencias Básicas de la Salud. Los jefes de departamento y coordinadores de carrera del área de la salud trabajaron en diciembre del 2007 y enero del 2008, un plan estratégico de desarrollo con presupuesto para 5 años. ${ }^{(2)}$

Para trabajar la viabilidad y factibilidad de creación de la Escuela, el Presidente de la Comisión de Transición activó el Grupo Gestor de la EUCS, equipo que realizó varias actividades dirigidas al desarrollo de conocimientos, actitudes y prácticas de organización y funcionamiento de la Escuela, por lo que se plantea que la EUCS de la UNAH-VS para dar respuesta a las exigencias del siglo XXI contará con un currículo orientado al enfoque humanístico-social, fundamentado en valores, desarrollando competencias en salud, fomentando el trabajo en equipo y privilegiando la investigación como herramienta de crecimiento académico, y proyectos de vinculación universidad-sociedad para enriquecer la docencia, responder a los problemas de la sociedad y cumplir con la finalidad de la UNAH.

\author{
Integrantes del Proyecto de la Escuela Univer- \\ sitaria de Ciencias de la Salud del 2006-2008: \\ Director de la UNAH-VS. \\ MSc. Carlos Alberto Pineda Fernández \\ Grupo Gestor de la Escuela Universitaria de \\ Ciencias de la Salud \\ MSc. Noemí Mendoza, \\ Jefa del Departamento de Enfermería. \\ MSc. Norma Urrutia, \\ Coordinadora de la Carrera de Enfermería. \\ Dra. María del Carmen Sauceda, \\ Jefa del Departamento de Psicología. \\ Dr. Francisco Herrera, Coordinador \\ de la Carrera de Medicina/Odontología. \\ Dr. Edwin Cruz, Comisión de Gestión \\ de la Carrera de Odontología. \\ Dr. José Antonio García, Comisión \\ de Gestión de la Carrera de Odontología. \\ MSc. Melba Baltodano, Comisión \\ de Gestión de la Carrera de Odontología.
}

MSc. Elba Rubí Morán, Comisión de Gestión de la Carrera de Odontología.

Dr. Reniery España, Coordinador

de la Maestría de Salud Pública.

Dr. Benjamín Abdú, Coordinador

Especialidad de Ginecología y Pediatría.

Coordinadores del Grupo Gestor de la Escuela Universitaria de Ciencias de la Salud

Dr. Francisco J. Herrera A.

MSc. Melba E. Baltodano.

MSc. Elba Rubí Morán.

Asesores Externos a las Ciencias de la Salud Lic. Aníbal Delgado Fiallos.

MSc. Brenda Hulse.

MSc. Carlos Alberto Pineda. ${ }^{(3)}$

Marco Conceptual, Contextual y Legal de la EUCS

El propósito de la EUCS es aportar con visión prospectiva a la construcción de conocimiento en salud, que permita la modificación de las prácticas de atención con la finalidad de contribuir a la transformación de la situación de salud del país y en particular en la región nor-occidental. La Escuela Universitaria de Ciencias de la Salud visualiza los problemas de salud de manera integral, es decir que busca la explicación multicausal, analizando los determinantes y condicionantes del proceso salud-enfermedad desde el punto de vista de la Biología Humana, los estilos y modos de vida, las condiciones ambientales y el rol de los servicios de salud. Para la toma de decisiones y gestión en salud utiliza herramientas que generan información valida y confiable como es el análisis de situación de salud, la vigilancia de la salud pública y la investigación en salud, considerando en todas ellas el enfoque holístico.

La Ciencia de la Salud se organiza en dos vertientes: el estudio y la investigación para la adquisición de conocimientos sobre el proceso salud-enfermedad y la aplicación de estos conocimientos técnicos. Ambas vertientes se reúnen para lograr el amplio propósito de: mantener, reponer y mejorar la salud y el bienestar; prevenir, tratar, rehabilitar y erradicar enfermedades y comprender mejor los procesos vitales de los humanos relacionados con la vida, la salud y sus alteraciones. 
El Modelo de la UNAH-VS en el análisis situacional de su entorno presenta que la Zona Metropolitana del Valle de Sula (ZMVS) tiene una extensión territorial de 7,600 kilómetros cuadrados y una población equivalente al $25 \%$ del total nacional, que corresponde al área de influencia de la EUCS, constituida por veinte municipios; los doce del departamento de Cortés, cuatro de Yoro: El Progreso, Santa Rita, Morazán y El Negrito, tres de Santa Bárbara: Quimistán, Las Vegas y Petoa, y uno de Atlántida: Tela. ${ }^{(3)}$

La fundamentación legal de la EUCS se encuentra en los preceptos de la Constitución de la República, Ley Orgánica de la UNAH y su reglamento, la Ley de Educación Superior y su reglamento, las Normas Académicas de la Educación Superior, y en otros instrumentos programáticos emitidos por la Comisión de Transición en su condición de depositaria de las atribuciones del Consejo Universitario y de la Junta de Dirección Universitaria.

La Constitución de la República en lo relacionado con el proyecto de la EUCS señala que le corresponde al Estado participar en la promoción y preservación de la salud personal y de la comunidad; que la educación es función esencial del Estado y que la UNAH tiene exclusividad de organizar, dirigir y desarrollar la educación superior y profesional.

La Ley Orgánica de la Universidad Nacional Autónoma de Honduras establece como estructura organizacional a la UNAH en Facultades, Centros Universitarios y Centros Regionales Universitarios, Departamentos, Carreras, e Institutos, uno de sus objetivos es fomentar la creación de Escuelas, Carreras, Institutos, consultorios y en general centros de estudios e investigación para facilitar la ampliación y profundización del conocimiento científico en el área respectiva (Ley Orgánica artículo \# 28 y su Reglamento General, artículo 78), La creación de la EUCS responde a los objetivos del plan de desarrollo de la UNAH, plan plurianual 2006- 2008, al Modelo Educativo de la UNAH y al Modelo Teórico de la UNAHVS. ${ }^{(3)}$
La Comisión de Transición de la UNAH según acta No. 169-2008 de la sesión celebrada el 12 de febrero del 2008 mediante el Acuerdo No. 348-A-2008 acuerda aprobar la creación de la Escuela Universitaria de Ciencias de la Salud (EUCS), adscrita a la Universidad Nacional Autónoma de Honduras en el Valle de Sula realizándose el lanzamiento de creación el 25 de abril del mismo año. (4) Las Carreras que la integran actualmente son: Enfermería, Odontología, Medicina, las especialidades de Pediatría y Gineco-obstetricia. La autoevaluación de las mismas, se realiza periódicamente, utilizando las guías e instrumentos que proponga el Sistema de Autoevaluación para la Acreditación de la UNAH, actualmente las 5 carreras se encuentran autoevaluadas contando con su informe respectivo e implementando el plan de mejora.

La EUCS se rige por el reglamento de las Escuelas Universitarias de la Universidad Nacional Autónoma de Honduras (UNAH) que fue aprobado según Acuerdo número CU-O-02502-2012, adoptado por el Consejo Universitario en su Sesión Ordinaria celebrada el día viernes 24 de febrero de 2012, contenido en el Acta número: CU-0-002-02- 2012 y publicado en La Gaceta el 14 de mayo de ese mismo año.(5)

Creación, conformación de nuevos departamentos y cambio de nombre de algunos ya existentes.

En el proyecto de creación de la Escuela de las Ciencias de la Salud, se contempla:

La creación de los siguientes Departamentos:

- Salud Pública.

- Patología.

- Medicina Clínica Integral.

El cambio de nombre de los Departamentos ya existentes:

- Odontología por el de Departamento de Ciencias Odontológicas.

- Enfermería por el de Departamento de Ciencias de la Enfermería.

- Morfológicas y Fisiológicas por el Departamento de Ciencias Básicas de la Salud. (3) 
En mayo 2008 se conforma el Depto. de Ciencias Básicas de la Salud, ocupando la primera Jefatura el Dr. José Raúl Arita Chávez, en febrero del 2012 se conforma el Depto. de Medicina Clínica Integral, ocupando la primera Jefatura el Dr. Marco Antonio Molina Soto, en mayo del 2014 se conforma el Depto. de Salud Pública, ocupando la primera Jefatura el Dr. Marvin Rodríguez Moreno. El área de Patología está adscrita a Ciencias Básicas.

Actualmente la EUCS cuenta con una planta docente de 102 profesores permanentes, 60 profesores por hora y 10 administrativos.

\section{Primeras Autoridades del Consejo Consul- tivo de la EUCS, 2008 - 2011:}

Dr. Francisco José Herrera Alvarado, Jefatura EUCS.

Licda. Eda Nohemi Mendoza,

Jefatura Depto. Ciencias de la Enfermería.

Licda. Norma Urrutia,

Coordinación Carrera de Enfermería.

Dra. Karla Rápalo,

Coordinación Carrera de Odontología.

Dr. Edwin Cruz,

Jefatura Depto. de Ciencias Odontológicas.

Dr. José Raúl Arita Chávez, Jefatura

Depto. de Ciencias Básicas de la Salud.
Dr. Benjamín Abdu, Coordinación

Especialidad de Ginecología y Obstetricia.

Dr. Enrique Jovel,

Coordinación Especialidad de Pediatría.

Con la Aprobación del reglamento de las Escuelas Universitarias en el 2012 pasa a llamarse Consejo Directivo, presidido por la Dirección de la Escuela. ${ }^{(6)}$ El Dr. Herrera Alvarado, fungía también durante este período como Coordinador de la Carrera de Medicina teniendo a su cargo el área clínica. El área de Salud Pública y Patología estaban adscritas al Departamento de Ciencias Básicas de la Salud que hasta el 2013 estuvo a cargo del Dr. Arita Chávez, quien en febrero del 2014 ocupa el cargo de Director de la EUCS. Hasta la actualidad se ha logrado avanzar de manera significativa en la conformación y consolidación de la estructura organizativa y funcional de la misma, con el apoyo de las autoridades locales superiores como ser: el Lic. Carlos Pineda Fernández como Exdirector y el Señor Director actual de la UNAH-VS Dr. Francisco J. Herrera A. ${ }^{(7,8)}$ El año 2012 se obtuvo una matrícula de 3822 estudiantes, siendo el mayor número hasta la fecha, (Ver Gráfica No. 1), los profesionales graduados en 2008 fueron 58 y el año 2015 fueron 181. Ver tabla No. 1.

Gráfica No. 1: Matrícula EUCS primer período de cada año, 2008-2016

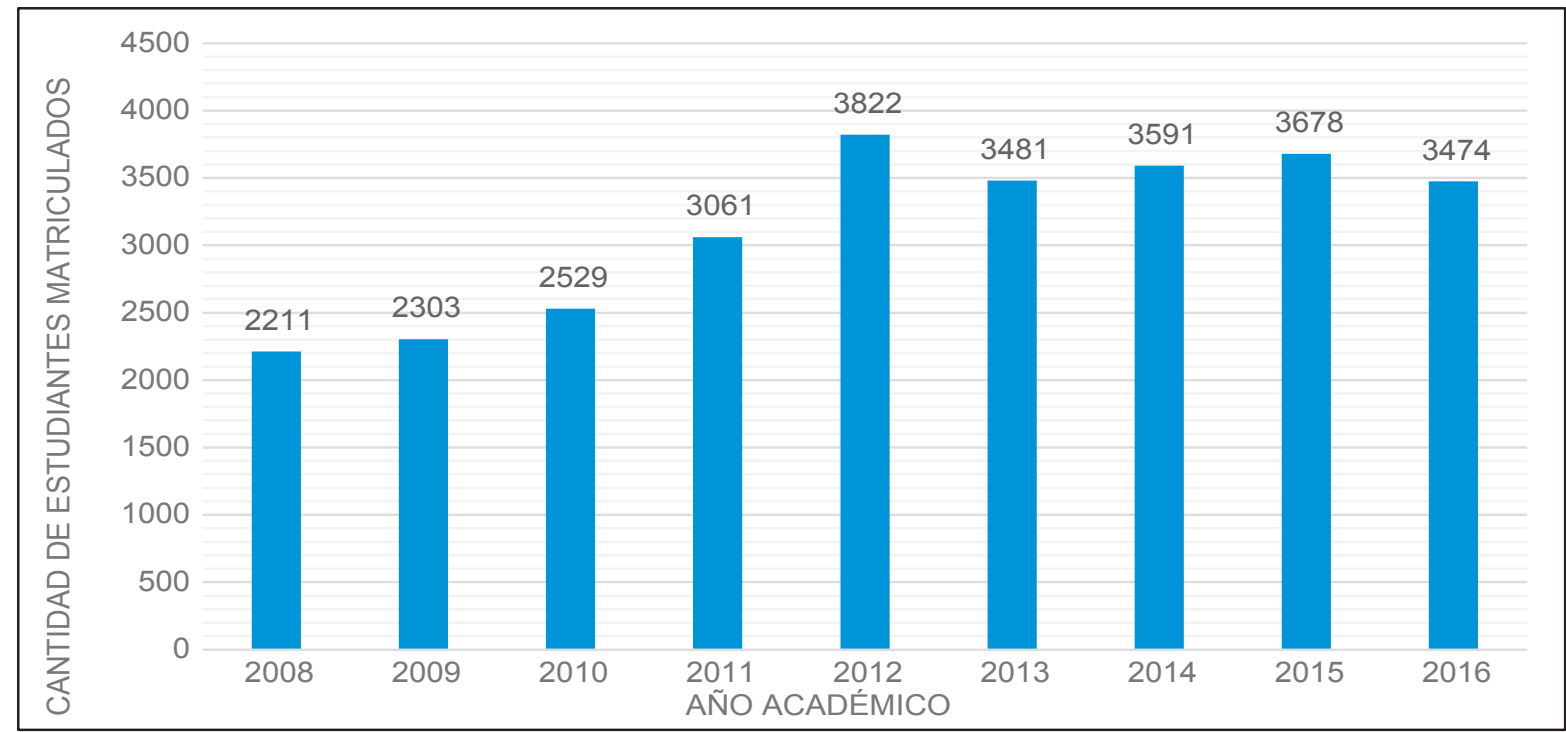

Fuente: Dirección de Ingreso Permanencia y Promoción (DIPP) de la UNAH. ${ }^{(7,8)}$

Se incluyen las carreras de Enfermería, Odontología, Medicina, Especialidad de Pediatría y Gineco-obstetricia. 
Tabla No. 1: Graduados de las cinco carreras de la EUCS del 2008 al 2015

\begin{tabular}{|c|c|c|c|c|c|c|c|c|c|}
\hline Carrera & $\mathbf{2 0 0 8}$ & $\mathbf{2 0 0 9}$ & $\mathbf{2 0 1 0}$ & $\mathbf{2 0 1 1}$ & $\mathbf{2 0 1 2}$ & $\mathbf{2 0 1 3}$ & $\mathbf{2 0 1 4}$ & $\mathbf{2 0 1 5}$ & Total \\
\hline Medicina & 0 & 0 & 0 & 0 & 0 & 29 & 71 & 83 & $\mathbf{1 8 3}$ \\
\hline Odontología & 18 & 29 & 32 & 71 & 78 & 76 & 82 & 59 & $\mathbf{4 4 5}$ \\
\hline Enfermería & 23 & 32 & 21 & 33 & 24 & 11 & 21 & 20 & $\mathbf{1 8 5}$ \\
\hline $\begin{array}{c}\text { Ginecología } \\
\text { y Obstetricia }\end{array}$ & 9 & 9 & 14 & 11 & 6 & 4 & 8 & 9 & $\mathbf{7 0}$ \\
\hline Pediatría & 8 & 5 & 4 & 10 & 9 & 7 & 15 & 10 & $\mathbf{6 8}$ \\
\hline Total & $\mathbf{5 8}$ & $\mathbf{7 5}$ & $\mathbf{7 1}$ & $\mathbf{1 2 5}$ & $\mathbf{1 1 7}$ & $\mathbf{1 2 7}$ & $\mathbf{1 9 7}$ & $\mathbf{1 8 1}$ & $\mathbf{9 5 1}$ \\
\hline
\end{tabular}

Fuente: Secretaría de la UNAH-VS y archivos de la EUCS.

La EUCS cada semestre desarrolla la Feria Científico-Cultural (Ver Figura No. 1) para contribuir a dar respuesta a su misión y visión, al artículo 2 de la Ley Orgánica de la UNAH, al modelo educativo de la UNAH, al modelo teórico de la UNAH-VS y así mismo a lo esencial de la reforma universitaria, incorporando ciencia, ética, arte, cultura y deporte, con la participación de autoridades, docentes y estudiantes de las diferentes carreras que integran la Escuela, así como la comunidad universitaria en general. ${ }^{(9)}$

Figura No. 1: Feria Científico Cultural de la EUCS, Primer Semestre 2013

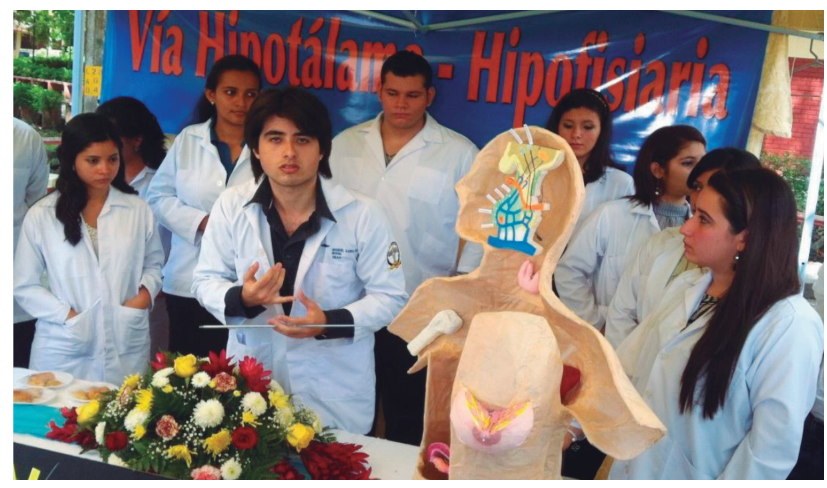

Estudiantes de Ciencias Morfológicas en presentación del tema.

\section{REVISTAS DE LA EUCS}

La EUCS promueve la elaboración, periodicidad y cumplimiento de todos los criterios internacionales para la indexación en bases de datos internacionales de dos Revistas Científicas que se publican actualmente:

1 Revista Científica de la Escuela Universitaria de las Ciencias de la Salud y 2) Acta Pediátrica Hondureña. Lo que sirvió de base esencial para solicitar la asignación respectiva y fundamental del ISSN (International Serial Estándar Number). Hasta la fecha, se han elaborado y divulgado varios números de los cuales tres de cada revista cuentan con ISSN, requisito para el proceso de indexación. Las versiones en línea son difundidas a través de la www.bvs.hn desde el año 2014.

\section{Revista Científica de la Escuela Universita- ria de las Ciencias de la Salud.}

(ISSN: versión impresa 2409-9759, en línea 2411-6289)

La EUCS en el año 2011 inició a trabajar con el consejo editorial de la revista de Ciencias de la Salud y se publicó un número correspondiente al primer semestre de ese año. En el año 2014 se reorganiza y se conforma el Consejo Editorial con representantes de los cinco departamentos que hasta la actualidad integran la Escuela, decidiendo cambiar el nombre a: Revista Científica de la EUCS, debido a que la periodicidad del primer número había sido interrumpido por más de dos años.

La Revista Científica de la EUCS, es el instrumento u órgano integrador oficial de difusión de trabajos científicos que promueve el desarrollo de la investigación, conocimiento científico, análisis y debate de la situación de salud, para mejorar las condiciones de vida de la población con el propósito de contribuir al desarrollo humano sostenible de nuestro país. Tiene una periodicidad de publicación semestral, apegada a las recomendaciones para la realización, presentación de informes, edición y publicación de trabajos académicos en revistas médicas, 
versión 2013, disponible en: http// www.ICMJE.org, elaborado por el Comité Internacional de Editores de Revistas Médicas.

\section{Primer Consejo Editorial: año 2014-2015: \\ Dirección: \\ Dra. Tania Soledad Licona Rivera \\ Comité de Edición: \\ Licda. Vilma Mercedes Miranda Vaquedano \\ Dra. Lourdes Azucena Andrade Ávila \\ Dr. Gustavo Ramos \\ Comité de Redacción: \\ Dra. Karen Erazo \\ Licda. María del Carmen Maldonado \\ Dr. Joel Ricardo Chávez Gutiérrez \\ Dr. Tulio Arnaldo Bueso Jacquier \\ Comité de Administración: \\ Dr. José Raúl Arita Chávez \\ Secretaría: \\ Dr. Carlos Amílcar Fuentes Romero}

Actualmente el Consejo Editorial fue reconformado con rotación de docentes de algunos departamentos para el periodo 2016-2017, siempre bajo la dirección de la Dra. Tania Soledad Licona Rivera. La Revista cuenta con su reglamento que fue elaborado por el primer Consejo Editorial, al inicio de sus funciones, revisado y aprobado por la Dirección de la EUCS, en junio del 2014. ${ }^{(10)}$

\section{Revista Científica: Acta Pediátrica Hondu-} reña. (ISSN: versión impresa 2410-1400, versión en línea 2411-6270)

La revista Acta Pediátrica Hondureña $(\mathrm{APH})$ es el órgano oficial de difusión del posgrado de Pediatría de la EUCS/UNAH-VS con periodicidad de publicación semestral.

Se considerará para publicación los escritos médicos relacionados con la salud de los niños, que cumplan con los requisitos de uniformidad para manuscritos enviados a revistas biomédicas elaborados por el Comité Internacional de Directores de Revistas Médicas (ICMJE). Disponible en: www.ICMJE.org.
Se inició a editar desde finales del año 2009, con la participación de los pediatras docentes del posgrado y el apoyo de los médicos asistenciales que brindan su labor ad-honoren, quienes mediante el aporte de las Casas Farmacéuticas, logran sufragar todos los gastos de impresión. APH llega a manos de médicos internos, en servicio social, médicos generales y especialistas quienes la utilizan para mantenerse informados sobre temas de actualidad, casos clínicos de escasa presentación y otros, ya que es distribuida por los visitadores médicos de manera gratuita en diferentes partes del país. En el año 2014, la EUCS integra un representante para velar por el cumplimiento de los criterios de indexación y fortalecer el Consejo Editorial.

\section{Primer Consejo Editorial: año 2009-2013:}

\section{Cuerpo Editorial:}

Editor en Jefe:

Dr. Julio Cesar Ortega Iglesias.

Comité Editorial:

Dra. Karen Sobeyda Erazo

Dra. Marlin del Carmen Erazo

Dra. Erika Elena Cole

Dra. Sandra Fiallos

Dr. Gabriel Enrique Bennett

Dr. Luis Enrique Jovel López

Dr. Oscar Gerardo Banegas

El Consejo Editorial es conformado con rotación de Médicos Residentes, docentes y Pediatras de los diferentes hospitales (Hospital Mario Catarino Rivas, Instituto Hondureño del Seguro Social y Hospital Leonardo Martínez). Desde el año 2014 la dirección está a cargo de la Dra. Lidia María Prado López. ${ }^{(10)}$

Actualmente, los contenidos de ambas revistas son divulgados en diferentes bases de datos. $Y$ según las gestiones realizadas, se espera que para el segundo semestre del 2016, sean indexadas en: Sistema Regional de Información en Línea para Revistas Científicas de América Latina, el Caribe, España y Portugal (Latindex) y Literatura Latinoamericana en Ciencias de la Salud (LILACS). 


\section{Internacionalización}

Septiembre - Octubre 2012: Proyecto de Movilidad: "XXIX Congreso Nacional de Anatomía" participación de cuatro docentes del Departamento de Ciencias Básicas de la Salud de la EUCS que se realizó en la Universidad Autónoma de Zacatecas, México. En este evento se logró la inscripción como miembros de la Sociedad Mexicana de Anatomía y la Asociación Panamericana de Anatomía con el propósito de continuar participando y crear los enlaces internacionales para fortalecer los intercambios académicos.

Julio 2012: Proyecto de movilidad: "Entrenamiento en laboratorio de prótesis dental" participación de trece estudiantes de la carrera de odontología en Daegu, Korea (Daegu Health College) permaneciendo por espacio de 30 días para tomar un entrenamiento en laboratorio de prótesis dental. ${ }^{(11)}$

Noviembre 2012: Proyecto de movilidad: "Firma de dos convenios de cooperación" Participación del Dr. Francisco Herrera como Director de la EUCS y Dr. Edwin Cruz como autoridad del departamento de Ciencias Odontológicas, en la firma de los convenios con Yonsei University, Seul, Korea y Daegu Health College, Daegu Korea con la misión de fortalecer la creación de posgrados y carreras cortas en la EUCS de la UNAH-VS. ${ }^{(11)}$

Septiembre - Octubre 2013: Proyecto de Movilidad: "XVII Congreso Panamericano de Anatomía" participación de cuatro docentes del Departamento de Ciencias Básicas de la Salud de la EUCS que se realizó en Huatulco, Oaxaca, México.

Mayo del 2014: Proyecto de Movilidad "Fortalecimiento del desarrollo de competencias docentes y de investigación en el área de la salud entre Universidades de la región" (Ver Figura No. 2) del Programa de Apoyo a la Integración Regional Centroamericana (PAIRCA II) y la Secretaria General Consejo Superior Universitario Centroamericano (CSUCA) por la EUCS de la UNAH-VS. Intercambio enfocado en los temas de Medicina, Odontología y Enfermería en reciprocidad de capacidades académicas, con énfasis en mecanismos de actualización y armonización mediante colaboraciones centroamericanas, el uso de nuevas tecnológicas y procesos innovadores de la adecuación de conocimientos a las demandas de cada sociedad; logrando así que las actividades de internacionalización permitan generar nuevas iniciativas regionales que potencien las funciones sustantivas de las universidades en lo que se refiere a la docencia, la investigación y la vinculación con la sociedad.

\section{Universidades Participantes:}

- Universidad Nacional Autónoma de Honduras en el Valle de Sula, UNAH-VS.

- Universidad de Costa Rica, UCR.

- Universidad de Panamá, UP.

\section{Equipo Académico participante:}

1. Francisco José Herrera Alvarado, Director de UNAH-VS.

2. José Raúl Arita Chávez, EUSC.

3. Marta Julia Amaya, Depto. de Ciencias de la Enfermería.

4. Emelda Ruth Suazo, Coordinación de Carrera de Enfermería.

5. Emilio Torres, Depto. Ciencias Odontológicas.

6. Roberto Pineda Leiva, Depto. de Ciencias Básicas de la Salud.

Apoyo de gestión, logística seguimiento e informe final del proyecto por la Licda. Alejandra Martínez como Coordinadora de Movilidad Académica en UNAH-VS. ${ }^{(12)}$

Figura No. 2: Intercambio de experiencias en la Universidad de Panamá, UP. Mayo 2014

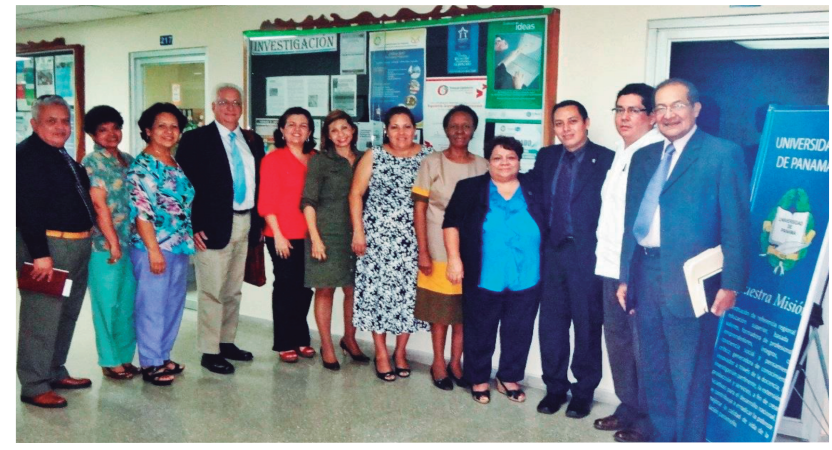

Equipo de Académicos de la EUCS y de la UP. 
Julio 2014: Proyecto de Movilidad: "Fortalecimiento del desarrollo de competencias docentes y estudiantiles en el manejo de la terminología morfológica internacional y el campo de investigación en el área de ciencias morfológicas" participación de ocho docentes y nueve estudiantes del Departamento de Ciencias Básicas de la Salud de la EUCS, de las diferentes carreras; Enfermería, Odontología y Medicina en el $10^{\circ}$ Simposio Ibero-latinoamericano de Terminología Anatómica, Embriológica e Histológica (SILAT X), que se realizó en Managua, Nicaragua, en el Campus de la Universidad Nacional Autónoma de Nicaragua. ${ }^{(13)}$

Agosto 2015: Proyecto Movilidad SILAT 2015, XII Simposio Ibero Latino America no de terminología Anatómica, Embriológica e Histológica, participación de ocho docentes y cuatro estudiantes del departamento de Ciencias Básicas de la Salud de la EUCS, de las diferentes carreras; Enfermería, Odontología y Medicina en el XII Simposio Ibero Latino Americano desarrollado en la Universidad de Costa Rica, a través del Colegio de Médicos y Cirujanos de la República de Costa Rica, de la Academia de Historia de la Medicina de Costa Rica, la Fundación de la Academia Panamericana de Historia de la Medicina. ${ }^{(14)}$

2014 - 2016: Proyecto de Movilidad: "Movilidad Académica Estudiantil "El papel de la UNAH a través de la Vicerrectoría de Relaciones Internacionales y la Vicerrectoría Académica participa en convocatorias para que la UNAH sea parte de los Consorcios Internacionales de becas Erasmus Mundus AMIDILA (Movilidad Académica para el Desarrollo de la Inclusión en América Latina) y EURICA (Europe and America: Enhancing University Relations by Investing in Cooperative Actions). En este marco la EUCS con el apoyo de la Coordinación de Movilidad Académica de la UNAH-VS, ha recibido a tres estudiantes extranjeros y ha enviado a varios estudiantes nuestros a otras universidades del extranjero, así como dos estudiantes próximamente a realizar movilidad en el segundo semestre de este año, tanto de grado como postgrado:
1. Luca Giudice de la Universidad de Padova Italia, pasante de IV año de la carrera de Medicina, cursó un semestre en la EUCS/ UNAH-VS, de julio a diciembre del año 2014. ${ }^{(12)}$

2. Cintia Alejandra Falck Fuentes de la UNAHVS, pasante del III año de la Especialidad de Pediatría, al Shriners Hospital for Chidren en Boston Estados Unidos durante el mes de octubre del 2014.

3. Federico Giordani de la Universidad de Padova Italia, pasante de $\mathrm{V}$ año de la carrera de Medicina, cursó un semestre en la EUCS/UNAH-VS, de julio a diciembre del año 2015.

4. Iveta Chmelová de la Universidad de Republica Checa, pasante de $\mathrm{V}$ año de la carrera de Fisioterapia, cursó un semestre en la EUCS/UNAH-VS, de julio a diciembre del año 2015. ${ }^{(13)}$

5. Melvin Eduardo Lagos García de la UNAH -VS, pasante de VI año de la carrera de Medicina, a la Universidad de Coimbra en Portugal, de septiembre del 2015 hasta febrero del año 2016.

6. Aylin Larissa Leiva Carbajal y Carlos Antonio Cubero Estevez de la UNAH-VS, pasantes de $\mathrm{V}$ año de la carrera de Medicina, para continuar y culminar sus estudios en la Universidad de Ishou, Taiwan a partir del segundo semestre de 2016.

7. Alejandra M. Valladares Ramos de la UNAH-VS, pasante del III año de la especialidad de Pediatría, al Instituto de Dermatología y Cirugía de Piel (INDERMA) en Guatemala, estará rotando durante el mes de septiembre del 2016.

8. Kelvin Flores Arévalo de la UNAH-VS, pasante del III año de la especialidad de Pediatría, al Hospital de Guadalajara Fray Antonio Alcalde en México, estará rotando durante el mes de septiembre del 2016.

\section{Investigación}

A nivel de grado se han realizado investigaciones en ciencias de la salud a lo interno de cada disciplina, desarrollando diferentes congresos para la presentación de sus resultados. A partir 
del presente año, los trabajos de investigación realizados por los estudiantes de último año de medicina como requisito de graduación, son asesorados y evaluados en la EUCS, para tal propósito se conformó la comisión coordinadora y asesora de los trabajos de investigación lo que fortalece esta actividad como función esencial del quehacer de la UNAH.

En los Posgrados; los trabajos de investigación para elaboración de la Tesis, son elaborados a lo largo de los tres años de residencia, con el acompañamiento de un asesor Metodológico y un asesor Técnico.

Desde el 2008 que egresaron los primeros médicos especialistas de la Escuela, se desarrolla el Congreso de Investigación Científica de los Posgrados avalado por el Centro de Educación Médica Continua (CENEMEC), del Colegio Médico de Honduras. La EUCS, mediante la Coordinación de investigación Científica en Posgrados a través de dicho evento promueve la actualización para estudiantes, médicos generales, especialistas y enfermeras del país.

Con el cumplimiento de la periodicidad ininterrumpida de la Revista Científica de la EUCS y Acta Pediátrica Hondureña, la comunidad estudiantil y docente de la Escuela ha sido motivada a desarrollar proyectos de investigación, que son difundidos en físico y en línea mediante dichas fuentes de publicación, otros trabajos han sido publicados en otras revistas nacionales e internacionales. En el marco de la Unidad de Gestión y del Comité de Ética de Investigación, actualmente se han conformado grupos de investigación inter y multidisciplinarios con interesantes propuestas de estudios.

\section{Vinculación}

Las carreras de las ciencias de la salud por su naturaleza y trabajo de campo en las diferentes comunidades o instituciones hospitalarias mantienen un aporte constante y sostenible de vinculación con la sociedad. Los estudiantes se desarrollan en escenarios de aprendizaje reales, logrando empoderarse de las competencias de manera integral al exponerse a la realidad de nuestro contexto. Algunas comunidades en donde se incursiona actualmente son Armenta, San Manuel Cortés y Gualjoco Santa Bárbara así como en otros municipios de la ZMVS.

Uno de los proyectos que es un reto para nuestra Universidad es la instalación y coordinación del Nodo Honduras, Campus Virtual en Salud Pública (CVSP), a través de la EUCS, en equipo con la Organización Panamericana de la Salud (OPS), la Secretaría de Salud (SESAL) y el País de Panamá, convirtiéndose este escenario en una oportunidad de fortalecimiento a la vinculación a través de alianzas estratégicas para brindar educación en todos los niveles por medio de esta plataforma en red internacional.

También la EUCS se vincula con la sociedad mediante la revista científica APH, que es distribuida de forma gratuita a nivel nacional en formato impreso a Médicos en Servicio Social, Generales y Especialistas en los diferentes hospitales del país sirviendo como instrumento de actualización en pediatría.

El proyecto ANGEL "Manos que salvan Vidas" como una iniciativa del Dr. Francisco Herrera presentada a la EUCS como eslabón inicial tienen la formación de una cultura preventiva, se enmarca en brindar capacitación a nuestros estudiantes, personal docente, administrativo y de servicios, mediante un taller teórico-práctico, enfocado al empoderamiento técnico de la Maniobra de Heimlich, planteando una línea de paralelismo proactivo, enfocada a la acción e intervención educativa para la salud, donde agentes y receptores de la misma se motivan ante la idea de brindar una respuesta eficaz, al encontrarse frente a la urgencia y emergencia de asfixia por obstrucción de la vía aérea por un cuerpo extraño (OVACE). La Dra. Ledezna Lizeth Arita como Coordinadora del proyecto, desde el 2014 hasta la actualidad ha trabajado en la elaboración, socialización y ejecución del mismo, contando con el apoyo de un equipo de especialistas y personal certificado en el tema, desarrollando 8 réplicas a diferentes grupos de 
participantes, con miras a extender estas buenas prácticas a la comunidad en general. ${ }^{(15)}$

\section{El complejo de la EUCS}

En julio del 2012 se coloca la primera piedra para la construcción del Complejo de la Escuela, (Ver Figura No. 3) con presencia de autoridades superiores de nuestra Universidad, Licda. Julieta Castellanos - Rectora de la UNAH, Lic. Carlos Pineda Fernández - Director de UNAH-VS, Dr. Francisco Herrera Alvarado Director de la EUCS y autoridades locales de San Pedro Sula Dr. Juan Carlos Zúniga - Alcalde Municipal; la obra ha avanzado de tal manera que pueda ser culminada a finales del 2016 y entrar en funcionamiento en el año 2017. La estructura arquitectónica del Complejo responde al enfoque integrador de los equipos de salud y funcionalmente traerá consigo un impacto significativo, ya que de manera positiva suplirá las necesidades de infraestructura y academia pertinente con calidad, que es necesaria en la formación de los futuros profesionales de las ciencias de la salud, así mismo la capacidad instalada en general brinda la posibilidad de ofrecer otras carreras afines, tanto de grado, postgrado como carreras técnicas, que en estas últimas se está trabajando en propuestas muy certeras, esto amplia las coberturas para los aspirantes universitarios.

Para nuestros docentes es importante ya que brinda los escenarios académicos, abundantes en laboratorios que son áreas de prácticas básicas indispensables, que permitirán fortalecer el eje de investigación científica así como la vinculación con la sociedad y promoción de la salud, alojando además las áreas administrativas para el funcionamiento efectivo general. Para la Zona Metropolitana del Valle de Sula significa un avance en educación superior, con aumento en las oportunidades de superación, y a medida la funcionalidad se consolide se ofrecerán algunos servicios específicos para apoyo a hospitales o instituciones de salud relacionadas y a la comunidad en general.

En el marco de los determinantes sociales de la salud y a través de la implementación de la Atención Primaria en Salud (APS), la Escuela tiene proyectado prestar servicios específicos orientados a la equidad en salud, con un enfoque eminentemente humanístico que a la vez contribuirán al desarrollo de las competencias cognitivas, procedimentales y actitudinales de nuestros estudiantes, al desarrollo de proyectos de investigación y vinculación con la sociedad favoreciendo a la disminución de los índices de morbi-mortalidad.

Los rubros planeados a la prestación de servicios son: atención odontológica, general médica, quirúrgica, servicio laboratorial y educación continua.

Figura No. 3: Colocación de la primera piedra del Complejo de la EUCS.

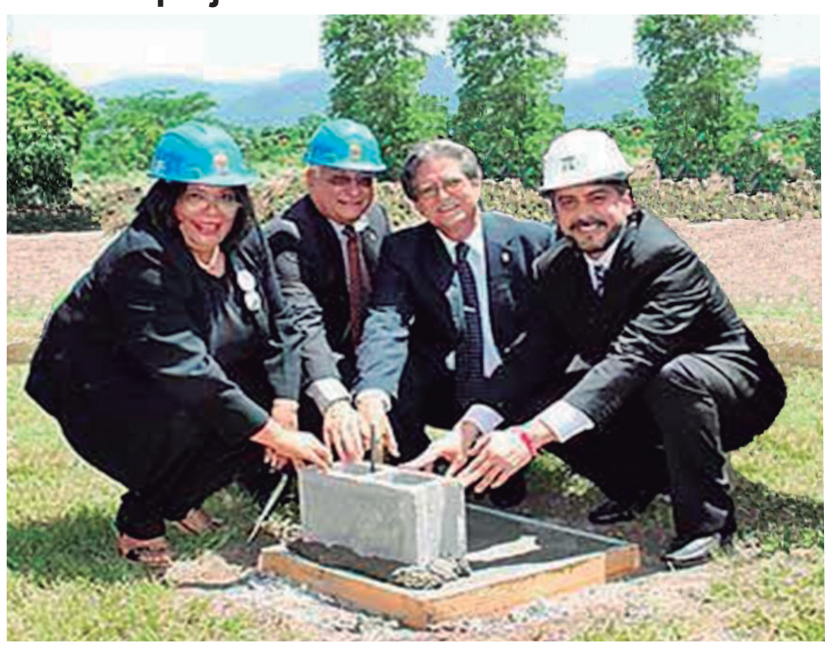

De Izquierda a Derecha: Licda. Julieta Castellanos, Dr. Francisco Herrera Alvarado, Lic. Carlos Pineda Fernández, Dr. Juan Carlos Zúniga.

\section{CONCLUSIÓN}

La EUCS es la unidad académica piloto como escuela universitaria a nivel nacional que sin duda alguna, rompe paradigmas y nos muestra distintos aprendizajes para el crecimiento en todos los ámbitos, personal, de gestión, docencia investigación y vinculación; ofreciendo nuevos retos que posicionan a nuestra Institución en niveles altos en educación superior enfocados a la globalización, contribuyendo de manera importante al desarrollo humano sostenible de nuestro país. 
Mi sincero agradecimiento por la confianza puesta en mí, de parte de las autoridades supe riores y de los compañeros y compañras, ya que es un honor estar actualmente a cargo de la dirección de tan compleja y distinguida labor académica.

\section{BIBLIOGRAFÍA}

1. Universidad Nacional Autónoma de Honduras en el Valle de Sula. Proyecto de creación de la Escuela Universitaria de Ciencias de la Salud. San Pedro Sula, Honduras, febrero 2008. Disponible en: https://histolo giaunahvs.files.wordpresscom/2011/03 leucs-proyecto. doc.

2. Moran Elba Rubí. Revista Ciencias de Salud, Escuela Universitaria de Ciencias de la Salud, UNAH-VS. 2011; 1(1):39-41.

3. Comisión de Transición UNAH, Vicerrectoría Académica UNAH. El Camino de la Reforma Universitaria, Fascículo No. 6, La Escuela Universitaria de Ciencias de la Salud de la UNAH-VS. Ciudad Universitaria, Tegucigalpa, Abril 2008.

4. Comisión de Transición de la UNAH. Acuerdo No. 348-A - 2008, acta No. 169-2008 CT del 2 de febrero del 2008, Ciudad Universitaria, Tegucigalpa, febrero 2008.

5. Consejo Universitario UNAH. Acuerdo número CU-O-025-02-2012, acta número: CU-O-002-02-2012 del 24 de febrero de 2012. La Gaceta el 14 de mayo del 2012, Tegucigalpa 2012.

6. Reglamento de las Escuelas Universitarias de la Universidad Nacional Autónoma de Honduras, La Gaceta, No. 32819, Republica de Honduras, Tegucigalpa M. D. C. 14 de Mayo del 2012.

7. Universidad Nacional Autónoma de Honduras en el Valle de Sula. Base de datos y estadísticas de la Escuela Universitaria de las Ciencias de la Salud, EUCS, UNAH-VS. Junio 2016.
8. Dirección de Ingreso Permanencia y Promoción de la Universidad Nacional Autónoma de Honduras. Datos estadísticos. Junio 2015.

9. Departamento de Ciencias Básicas de la Salud EUCS, UNAH-VS. Documento de La Feria Científico Cultural de la EUCS/ UNAHVS, San Pedro Sula, Abril 2012.

10. Licona Rivera TS, Arita Chávez JR. Informe de las Revistas de la Escuela Universitarias de las Ciencias de la Salud, UNAH-VS. Febrero del 2016.

11. Cruz Fajardo EF. Historia de Odontología en la Universidad Nacional Autónoma de Honduras en el Valle de Sula. Revista científica de la Escuela Universitaria de las Ciencias de la Salud. 2014; 1(2):9-10.

12. Coordinación de Movilidad Académica de la UNAH-VS. Informe Proyecto de Movilidad del Programa de Apoyo a la Integración Regional Centroamericana (PAIRCA II) y la Secretaria General Consejo Superior Universitario Centroamericano (CSUCA) por la Escuela Universitaria de las Ciencias de la Salud. San Pedro Sula, Julio 2014.

13. Escuela Universitaria de Ciencias de la Salud, UNAH-VS. Memoria Anual 2014. San Pedro Sula, noviembre 2014.

14. Escuela Universitaria de Ciencias de la Salud, UNAH-VS. Memoria Anual 2015. San Pedro Sula, noviembre 2015.

15. Escuela Universitaria de Ciencias de la Salud, UNAH-VS. Documento e Informe de El proyecto ANGEL "Manos que salvan Vidas". San Pedro Sula, julio 2014-junio 2016. 